\title{
Inverse magnetic catalysis in the soft-wall model of AdS/QCD
}

\author{
Danning Li, ${ }^{a}$ Mei Huang, ${ }^{b, c, d}$ Yi Yang ${ }^{e}$ and Pei-Hung Yuan ${ }^{f}$ \\ ${ }^{a}$ Department of Physics, Jinan University, \\ Guangzhou 510632, P.R. China \\ ${ }^{b}$ Institute of High Energy Physics, Chinese Academy of Sciences, \\ Beijing 100049, P.R. China \\ ${ }^{c}$ University of Chinese Academy of Sciences, \\ Beijing 100049, P.R. China \\ ${ }^{d}$ Theoretical Physics Center for Science Facilities, Chinese Academy of Sciences, \\ Beijing 100049, P.R. China \\ e Department of Electrophysics, National Chiao Tung University, \\ Hsinchu, R.O.C. \\ ${ }^{f}$ Institute of Physics, National Chiao Tung University, \\ Hsinchu, R.O.C. \\ E-mail: lidanning@jnu.edu.cn, huangm@ihep.ac.cn, \\ yiyang@mail.nctu.edu.tw, phy.pro.phy@gmail.com
}

ABSTRACT: Magnetic effects on chiral phase transition have been investigated in a modified soft-wall AdS/QCD model, in which the dilaton field is taken to be negative at the ultraviolet region and positive at the infrared region as in Phys. Rev. D 93 (2016) 101901 and JHEP 04 (2016) 036. The magnetic field is introduced into the background geometry by solving the Einstein-Maxwell system. After embedding the magnetized background geometry into the modified soft-wall model, the magnetic field dependent behavior of chiral condensate is worked out numerically. It is found that, in the chiral limit, the chiral phase transition remains as a second order at finite magnetic field $B$, while the symmetry restoration temperature and chiral condensate decrease with the increasing of magnetic field in small $B$ region. When including finite quark mass effect, the phase transition turns to be a crossover one, and the transition temperature still decreases with increasing magnetic field $B$ when $B$ is not very large. In this sense, inverse magnetic catalysis effect is observed in this modified soft-wall AdS/QCD model.

KEYwords: Holography and quark-gluon plasmas, Gauge-gravity correspondence, Phase Diagram of QCD

ARXIV EPRINT: 1610.04618 


\section{Contents}

1 Introduction 1

2 Gravity background 2

2.1 Equation of motion in EM system 3

2.2 Perturbative expansion in powers of $z \quad 4$

3 Magnetic field effects on chiral phase transition $\quad 7$

3.1 Inverse magnetic catalysis in the chiral limit $\quad 9$

$\begin{array}{lll}3.2 & \text { Inverse magnetic catalysis at finite quark mass } & 11\end{array}$

4 Conclusion and discussion $\quad 13$

$\begin{array}{ll}\text { A Perturbative solutions in powers of } B & 14\end{array}$

\section{Introduction}

Strong magnetic fields play essential roles in various physical systems, such as in the strong and weak phase transition of the universe $[1,2]$ and in noncentral heavy ion collisions at the Relativistic Heavy Ion Collider (RHIC) and the Large Hadron Collider (LHC) [36]. Besides the phenomenological importance, in theoretical aspects of strong interaction, strong magnetic fields also provide good probe of the dynamics of quantum chromodynamics(QCD), of which the vacuum structure are of numerous interests.

Spontaneous chiral symmetry breaking, characterized by the non-zero quark condensate $\sigma \equiv\langle\bar{\psi} \psi\rangle$, is one of the most representative features of QCD vacuum. At sufficient high temperature, it is believed that chiral symmetry would be restored when chiral condensate is destroyed by the temperature effect. To speculate the effect of magnetic fields on the behavior of chiral condensate in QCD at both zero and finite temperatures is of great interest for decades. Since the 1990's, enhancement of quark condensate and increasing of transition temperature under magnetic fields, known as the magnetic catalysis, has been recognized [7-10]. The latter studies from effective models and approximations to QCD [11-22], as well as lattice simulations [23-27] gave results in coincidence with these studies. Nevertheless, an inverse result showing that the transition temperature decreases with increasing magnetic field, known as the inverse magnetic catalysis(IMC), has just been discovered in recent lattice simulation [28, 29]. This surprising result is confirmed by latter lattice simulations [30]. Several efforts from different approaches [31-41] have been made to explain the microscopic mechanism of this phenomena.

In QCD, it is well known that the dominant physics related to chiral phase transition is non-perturbative, when the normal perturbative methods become invalid. Besides 
lattice simulation, the discovery of the anti-de Sitter/conformal field theory (AdS/CFT) correspondence and the conjecture of the gravity/gauge duality [42-44] offers a new powerful tool to solve the strong coupling problem in the relevant physical region. In dealing with chiral phase transition with magnetic fields, from top-down holographic approach, a kind of IMC effect has been realized in [45]. Instead of calculating the order parameter of chiral phase transition, the authors considered the phase transition by comparing the free energy of different geometric configuration. Similarly, [46] tried to investigate the phase transition temperature by comparing the free energy of different geometric backgrounds in bottom-up approach. They also found that the transition temperature decreases with increasing magnetic field. In some sense, it is a kind of IMC for deconfinement phase transition, since the geometric transition in bottom-up approach is usually considered as confinement/deconfinement phase transition. In other ways, the authors of [47] proposed a single scalar model coupled with a U(1) gauge field. After inserting the anomalous dimension into the scalar field, they observed the inverse magnetic catalysis behavior from chiral condensate, the order parameter of chiral phase transition.

Recently, from a more realistic bottom-up holographic framework, [48] tried to investigate the IMC effect within the soft-wall framework [49], which has been successfully applied and tested in describing hadron physics and relevant phenomena [50-63]. However, by simply extending the original soft-wall model and considering the magnetized geometric background from Einstein-Maxwell system [64-66], the magnetic field dependent chiral condensate showed that there is no IMC effect in the original soft-wall model by this scenario. Nevertheless, as noted in $[67,68]$, the background settings of original soft-wall model cannot describe spontaneous chiral symmetry breaking in the vacuum and its restoration at sufficient high temperature well. Thus, a modified version of soft-wall model was proposed in $[67,68]$. After introducing a modified dilaton field and extending the soft-wall model to finite temperature, chiral phase transition are realized perfectly. Therefore, it is interesting to study the magnetic field effects on chiral phase transition in this modified soft-wall model.

In this work, we will extend the study in $[67,68]$, and try to investigate how magnetic fields affect chiral condensate and chiral phase transition. The paper is organized as follows: in section 2, we will give a brief introduction on how to introduce the magnetic field from the Einstein-Maxwell system. In section 3, we extract the results of magnetic field dependent chiral condensate from the modified soft-wall model, both in the chiral limit and at finite quark mass. Finally, a short conclusion and discussion will be given in section 4 .

\section{Gravity background}

In $[67,68]$, the spontaneous chiral symmetry breaking in the vacuum and its restoration at finite temperature are realized perfectly in the soft-wall AdS/QCD model. There, the soft-wall model was put into the AdS-Schwarzchild(AdS-SW) black hole background as a probe. In these studies, only temperature effect and mass effect on chiral condensate are considered. To introduce the magnetic field, as was done in [46, 48], a simple way is to consider the gravity background with back-reaction of magnetic field through the 
Einstein-Maxwell(EM) system, with the action $^{1}$

$$
S_{B}=\frac{1}{16 \pi G_{5}} \int d^{5} x \sqrt{-g}\left(R-F^{M N} F_{M N}+\frac{12}{L^{2}}\right)
$$

where $R$ is the scalar curvature, $G_{5}$ is the 5 D Newton constant, $g$ is the determinant of metric $g_{M N}, F_{M N}$ is a $\mathrm{U}(1)$ gauge field, $L$ is the AdS radius and $M, N=0,1, \ldots, 4$.

Within this system, the authors of [46] compared the free energy of thermal AdS and black hole solution. In this way, they extracted the transition temperature and found that it would decrease with increasing magnetic field, showing a kind of inverse magnetic catalysis. As noted in the introduction, in some sense this kind of transition is related to confinement/deconfinement phase transition, since from the previous studies [69-77] the relevant order parameters to distinguish the two phases are the loop operators, which is related to confinement/deconfinement phase transition more closely. To be more concrete and limited in chiral phase transition, ref. [48] put the soft-wall model into the same metric background and extracted the magnetic dependent behavior of chiral condensate, which is the order parameter of chiral phase transition. They found that there is no inverse magnetic catalysis for chiral phase transition using this scenario. However, the simple version of soft-wall model cannot give well description of spontaneous chiral symmetry breaking even in the vacuum $[67,68]$. Thus, it is necessary to consider the magnetic effects in an improved version of soft-wall model. For simplicity, we will first consider the simple gravity background given by the Einstein-Maxwell system. The back-reaction from matter part(soft-wall action) to the metric will be neglected in this simple version. Before we move to deal with chiral condensate, in this section, we will first give a short introduction about the background gravity solution for preparation.

\subsection{Equation of motion in EM system}

The Einstein equation for the EM system could be easily found in the text books, and it takes the form

$$
E_{M N}-\frac{6}{L^{2}} g_{M N}-2\left(g^{I J} F_{M I} F_{N J}-\frac{1}{4} F_{I J} F^{I J} g_{M N}\right)=0,
$$

with $E_{M N}$ the Einstein tensor, defined in terms of the Ricci tensor $R_{M N}$ and Ricci scalar $R$ as $E_{M N}=R_{M N}-\frac{1}{2} R g_{M N}$. Here $M, N, I, J=0,1, \ldots, 4$.

The field equation for $F_{M N}$ is of the form

$$
\nabla_{M} F^{M N}=0
$$

and one can check that the constant magnetic field configuration

$$
F=\frac{B}{L} d x_{1} \wedge d x_{2}
$$

satisfies this equation.

\footnotetext{
${ }^{1}$ It is more rigorous to introduce magnetic field by considering the relation between electric charge, baryon number and $I_{3}$, the last two of which are related to the global symmetry of QCD. Here, we just follow $[46,48]$ and take the simplest version to introduce magnetic field.
} 
Since the magnetic field along $x_{3}$ axis breaks the rotation symmetry, the ansatz for the metric could be taken as

$$
d S^{2}=\frac{L^{2}}{z^{2}}\left(-f(z) d t^{2}+\frac{1}{f(z)} d z^{2}+h(z)\left(d x_{1}^{2}+d x_{2}^{2}\right)+q(z) d x_{3}^{2}\right) .
$$

For a black hole solution, $f\left(z=z_{h}\right)=0$ at horizon $z=z_{h}$ and $q(z)$ together with $h(z)$ are regular function of $z$ in the region $0<z<z_{h}$.

Under this metric ansatz and the magnetic field configuration eq. (2.4), the Einstein equations could be simplified as

$$
\begin{aligned}
& f^{\prime \prime}+\left(\frac{h^{\prime}}{3 h}+\frac{q^{\prime}}{6 q}-\frac{3}{z}\right) f^{\prime}+\left(-\frac{2 h^{\prime} q^{\prime}}{3 h q}-\frac{h^{\prime 2}}{3 h^{2}}+\frac{2 h^{\prime}}{z h}+\frac{q^{\prime}}{z q}\right) f-\frac{8 B^{2} z^{2}}{3 h^{2}}=0 \\
& q^{\prime \prime}+\left(\frac{2 f^{\prime}}{3 f}+\frac{h^{\prime}}{3 h}-\frac{2}{z}\right) q^{\prime}+\left(-\frac{2 f^{\prime} h^{\prime}}{3 f h}-\frac{h^{\prime 2}}{3 h^{2}}+\frac{2 h^{\prime}}{z h}-\frac{8 B^{2} z^{2}}{3 f h^{2}}\right) q-\frac{q^{\prime 2}}{2 q}=0
\end{aligned}
$$

and

$$
h^{\prime \prime}+\left(\frac{f^{\prime}}{3 f}-\frac{q^{\prime}}{6 q}-\frac{1}{z}\right) h^{\prime}+\left(-\frac{f^{\prime} q^{\prime}}{3 f q}+\frac{q^{\prime}}{z q}\right) h-\frac{h^{\prime 2}}{3 h}+\frac{4 B^{2} z^{2}}{3 f h}=0,
$$

together with a constrain equation

$$
\frac{f^{\prime} h^{\prime}}{2 f h}+\frac{f^{\prime} q^{\prime}}{4 f q}+\frac{h^{\prime} q^{\prime}}{2 h q}+\frac{h^{\prime 2}}{4 h^{2}}-\frac{3 f^{\prime}}{2 z f}-\frac{3 h^{\prime}}{z h}-\frac{3 q^{\prime}}{2 z q}-\frac{6}{z^{2} f}+\frac{6}{z^{2}}+\frac{B^{2} z^{2}}{f h^{2}}=0 .
$$

Within this system, the perturbative and full solutions of this system have been studied in several works $[64,65,80-82]$. The next subsection will be devoted to solve these equations and extract the metric background with magnetic field.

\subsection{Perturbative expansion in powers of $z$}

Requiring asymptotic AdS boundary condition, it is hard to get the exact analytical solution of eqs. (2.6)-(2.9). The full solution requires numerical method. Nevertheless, as given in [64], the leading expansion for these equations could be easily derived, taking the form

$$
\begin{aligned}
f(z) & =1+\frac{2}{3} B^{2} \ln (z / L) z^{4}+f_{4} z^{4} \\
q(z) & =1+\frac{2}{3} B^{2} \ln (z / L) z^{4} \\
h(z) & =1-\frac{1}{3} B^{2} z^{4} \ln (z / L)
\end{aligned}
$$

with $f_{4}$ the integral constants related to the black hole horizon $z_{h}$, where $f(z)=0$. Here, as pointed out in [65], $B$ is related with the physical magnetic field $\mathcal{B}$ at the boundary by the equation $\mathcal{B}=\sqrt{3} B$. Latter, for simplicity, we will consider $B$ instead of $\mathcal{B}$. We will see that the expansion could be a good approximation in the small $B$ and high temperature $T$ region. One can check that when $B$ goes to zero, it would reduce to the AdS-SW black hole solution. Thus, if the temperature and magnetic filed of interest are in this region, we can use this expansion as an approximation. Indeed, in [46, 48], the authors took this leading expansion as the metric background and studied the magnetic effects on the phase 
transition temperature. Since they only considered small magnetic field and in the final results, the relevant transition temperatures are not very low(in the relevant magnetic field region), the qualitative results are reliable. In this sense, because we are interested only in the region of near transition point and focus only in the qualitative results, we can follow these studies and only consider the perturbative expansion as first approximation.

Before we start to study the behavior of chiral condensate under magnetic fields, we would like to make more efforts on estimating the errors by using the perturbative solution eqs. (2.10)-(2.12). Firstly, we try to get perturbative solutions of eqs. (2.6)-(2.9) with more powers of $z$. Here, as an example, we list the solutions up to $z^{12}$. One can check the following near boundary expansion could satisfy eqs. (2.6)-(2.9) to $O\left(z^{13}\right)$

$$
\begin{aligned}
f^{(12)}(z)= & +\frac{2}{3} B^{2} \ln (\mu z) z^{4}+f_{4} z^{4} \\
& +B^{4}\left(-\frac{2}{63} \ln ^{2}(\mu z)+\frac{25}{441} \ln (\mu z)-\frac{181}{8232}\right) z^{8} \\
& +B^{4} f_{4}\left(\frac{5}{231} \ln ^{2}(\mu z)-\frac{1003}{106722} \ln (\mu z)+\frac{209015}{131481504}\right) z^{12} \\
& +B^{6}\left(\frac{10}{693} \ln ^{3}(\mu z)-\frac{2773}{640332} \ln ^{2}(\mu z)+\frac{626341}{197222256} \ln (\mu z)-\frac{12782437}{17355558528}\right) z^{12} \\
& +O\left(z^{16}\right) \\
q^{(12)}(z)= & +\frac{2}{3} B^{2} \ln (\mu z) z^{4} \\
& -B^{2} f_{4}\left(\frac{1}{3} \ln (\mu z)+\frac{1}{24}\right) z^{8}+B^{4}\left(-\frac{2}{63} \ln ^{2}(\mu z)+\frac{25}{441} \ln (\mu z)-\frac{181}{8232}\right) z^{8} \\
& +B^{2} f_{4}^{2}\left(\frac{2}{9} \ln (\mu z)+\frac{1}{27}\right) z^{12}+B^{4} f_{4}\left(\frac{10}{99} \ln ^{2}(\mu z)-\frac{148}{3267} \ln (\mu z)+\frac{20051}{1724976}\right) z^{12} \\
& +B^{6}\left(\frac{10}{693} \ln (\mu z)-\frac{2773}{640332} \ln ^{2}(\mu z)+\frac{626341}{197222256} \ln (\mu z)-\frac{12782437}{17355558528}\right) z^{12} \\
& +O\left(z^{16}\right) \\
& 1-\frac{1}{3} B^{2} z^{4} \ln (\mu z) \\
& +B^{2} f_{4}\left(\frac{1}{6} \ln (\mu z)+\frac{1}{48}\right) z^{8}+B^{4}\left(\frac{17}{126} \ln ^{2}(\mu z)-\frac{47}{1764} \ln (\mu z)+\frac{305}{32928}\right) z^{8} \\
& -B^{2} f_{4}^{2}\left(\frac{1}{9} \ln (\mu z)+\frac{1}{54}\right) z^{12}+B^{4} f_{4}\left(-\frac{35}{198} \ln ^{2}(\mu z)+\frac{415}{52272} \ln (\mu z)-\frac{17131}{3449952}\right) z^{12} \\
& +B^{6}\left(-\frac{101}{1386} \ln { }^{3}(\mu z)+\frac{28939}{1280664} \ln ^{2}(\mu z)-\frac{590707}{65740752} \ln (\mu z)+\frac{38983919}{104133351168}\right) z^{12} \\
& +O\left(z^{16}\right) \\
h^{(12)}(z) & (2.15 \\
&
\end{aligned}
$$

In the above expressions, we have two integral constants $f_{4}$ and $\mu$. If one takes $\mu=\frac{1}{L}$, then the leading expansion would be exactly the one in eqs. (2.10)-(2.12). However, for a black hole solutions, these two integral constants are not independent, due to the near horizon boundary condition. Usually, the near horizon boundary condition are required as the regular condition of the equation of motion. The definition of the horizon $z_{h}$ is $f\left(z_{h}\right)=0$, so in eqs. (2.7), (2.8) apparent singularity appears where $f(z)$ arises as the denominators. If we collect such kind of terms out, we find that in eq. (2.7) and eq. (2.8) 


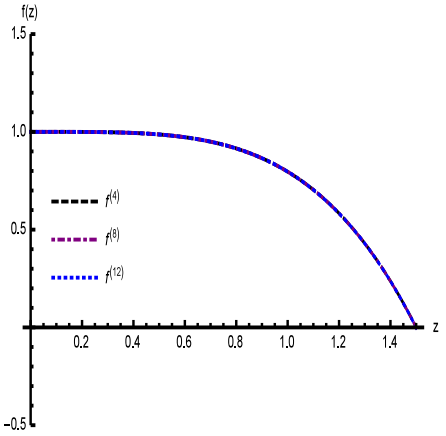

(a)

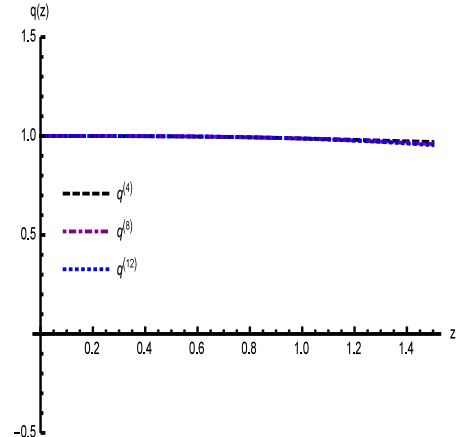

(b)

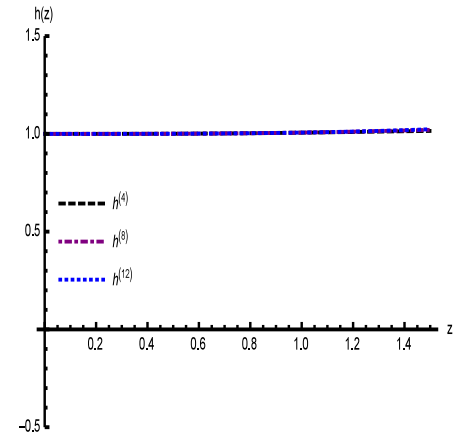

(c)

Figure 1. Different orders of functions $f(z), q(z), h(z)$ as functions of $z$ when $B=0.15 \mathrm{GeV}^{2}, \mu=$ $0.45 \mathrm{GeV}, z_{h} \simeq 1.5 \mathrm{GeV}^{-1}$, which gives $T \simeq 208 \mathrm{MeV}$.

they takes the form $\frac{Q(z)}{f(z)}$ and $-2 \frac{q(z)}{h(z)} \frac{Q(z)}{f(z)}$, with

$$
Q(z) \equiv-\frac{8 B^{2} z^{2} q}{3 h^{2}}-\frac{2 q f^{\prime} h^{\prime}}{3 h}+\frac{2}{3} f^{\prime} q^{\prime}
$$

Since we require the solutions to be regular at horizon, there would be a natural regular condition

$$
Q\left(z_{h}\right)=0
$$

to cancel the $f\left(z_{h}\right)=0$ singularity at horizon. This regular condition would require $\mu$ to depend on $f_{4}$. In this way, there are no free parameters in the full solutions. In general, when $B$ is fixed, $\mu, f_{4}$ would be a function of temperature $T$. Thus, the full solutions would be fixed when $B, T$ are given.

From the structure of the UV expansion, the leading, next-leading, next-next-leading powers are of $z^{4}, z^{8}, z^{12}$ respectively. In between those terms, there are no powers like $z^{5}, z^{6}, z^{7}, z^{9}, z^{10}, z^{11}$. We would expect that the leading expansion could provide good approximation in small $z$ area. Thus, if $z_{h}$ is very small, we could expect that the perturbative solution could be used in the whole region from boundary to horizon. As pointed out in [46], the perturbative solution could work well when $B \ll T^{2}$. To be more explicitly, we plot the functions $f^{(4)}, q^{(4)}, h^{(4)}, f^{(8)}, q^{(8)}, h^{(8)}, f^{(12)}, q^{(12)}, h^{(12)}$ to show the errors for small $T$ and large $T$ in figure 1 and figure 2 . In figure 1 , we take $B=0.15 \mathrm{GeV}^{2}, \mu=0.45 \mathrm{GeV}, z_{h} \simeq 1.5 \mathrm{GeV}^{-1}$, which gives $T \simeq 208 \mathrm{MeV}$. We could see that in Panel(a),(b),(c), the three lines for perturbative expansions to different orders are almost the same, showing good convergence of the perturbative expansion in this value of magnetic field and temperature. Simultaneously, in figure 2, we plot the corresponding functions when $B=0.15 \mathrm{GeV}^{2}, \mu=0.45 \mathrm{GeV}, z_{h} \simeq 3.5 \mathrm{GeV}^{-1}$ and $T \simeq 40 \mathrm{MeV}$. There we could see that the convergence of the perturbative expansion becomes very bad. $h^{(12)}(z)$ is even negative within $0<z<z_{h}$, showing the invalidity of the expansion solution in this physical region, where a full numerical solution is necessary.

Thus, if considering a temperature region not very low, we can take the leading expansion as a first test to catch the qualitative behavior, instead of trying to solve the full 


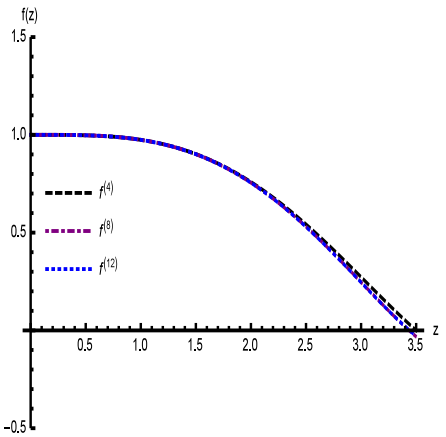

(a)

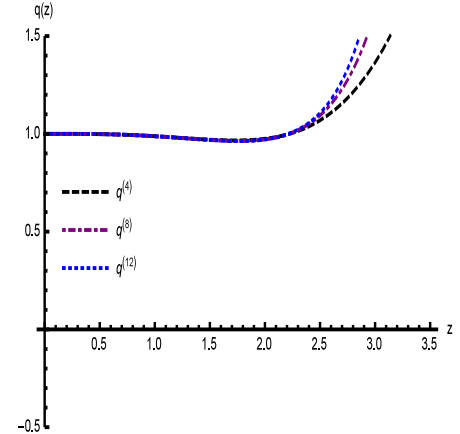

(b)

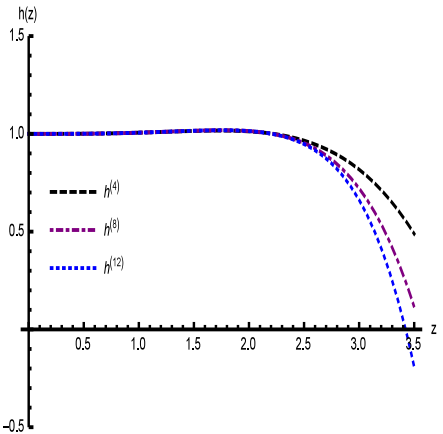

(c)

Figure 2. Different orders of functions $f(z), q(z), h(z)$ as functions of $z$ when $B=0.15 \mathrm{GeV}^{2}, \mu=$ $0.45 \mathrm{GeV}, z_{h} \simeq 3.5 \mathrm{GeV}^{-1}$, which gives $T \simeq 40 \mathrm{MeV}$.

metric background. From the above discussion, we can expect that the small $B$ and large temperature results would be reliable, since the expansion solution could be good approximation to the full solutions in this corner. As a first approximation, we will only take the leading expansion

$$
\begin{aligned}
& f(z)=1-\frac{z^{4}}{z_{h}^{4}}\left(1+\frac{2}{3} B^{2} z_{h}^{4} \log \left(\frac{z}{z_{h}}\right)\right), \\
& q(z)=1+\frac{2}{3} B^{2} \ln (\mu z) z^{4} \\
& h(z)=1-\frac{1}{3} B^{2} z^{4} \ln (\mu z)
\end{aligned}
$$

in later calculation. In this expansion, magnetic field $B$ enters directly in the metric and the temperature is related to the horizon by

$$
T=-\frac{f^{\prime}\left(z_{h}\right)}{4 \pi}=\frac{1}{\pi z_{h}}-\frac{B^{2} z_{h}^{3}}{6 \pi} .
$$

Under this expansion, one can take proper values of $z_{h}$ and $B$ to set the temperature $T$ and magnetic field $B$ in the dual $4 \mathrm{D}$ theory. To be more careful, as in [46, 48], we will vary $\mu$ from $0.25 \mathrm{GeV}$ to $1 \mathrm{GeV}$ to check the convergence of the expansion and estimate the possible errors in the later calculation. ${ }^{2}$

\section{Magnetic field effects on chiral phase transition}

In the previous section, we have introduced the magnetic field into the metric background by solving the EM system. In this section, we will take the perturbative expansion eqs. (2.18)-(2.20) as the background geometric and consider its effect on chiral phase transition. We will extend the studies in $[67,68]$ to finite $B$ case and try to extract the

\footnotetext{
${ }^{2}$ But we emphasize that $\mu$ is not a free parameter. Instead, in principle, it could be solved by considering the near horizon boundary condition. However, when the perturbative expansion are valid, the difference of solutions with different $\mu$ is very small.
} 
magnetic effects on chiral phase transition in the framework holography. More concretely, we will embed the following soft-wall action in this perturbative metric background:

$$
S=-\int d^{5} x \sqrt{-g} e^{-\Phi} \operatorname{Tr}\left(D_{M} X^{+} D^{M} X+V_{X}+\frac{1}{4 g_{5}^{2}}\left(F_{L}^{2}+F_{R}^{2}\right)\right),
$$

with $A_{L / R}$ the left/right hand gauge field, $D_{M}$ the covariant derivative defined as $D_{M} X=$ $\partial_{M} X-i A_{M}^{L} X+i X A_{M}^{R}, F_{M N}$ the field strength defined as $F_{M N}=\partial_{M} A_{N}-\partial_{N} A_{M}-$ $i\left[A_{M}, A_{N}\right], V_{X}$ the scalar potential, $g_{5}$ the gauge coupling, $g$ the determinant of the metric, $\Phi$ the dilaton field and $M, N=0,1, \ldots, 4$. The leading term of $V_{X}$ is the mass term of the complex scalar field $X M_{5}^{2} X X^{+}$and $M_{5}^{2}$ can be determined as $M_{5}^{2}=-3$ (we take the AdS radius $L=1$ in this work) from the AdS/CFT prescription $M_{5}^{2}=(\Delta-p)(\Delta+p-4)$ [44] by taking $\Delta=3, p=0$.

As [48], we consider the simplest case and the magnetic field are taken into account only through the metric. We would not consider the possibility of higher order coupling between the magnetic filed and $X$ like in [47]. As in [49], we assume that the vacuum expectation value of $X$ field takes the diagonal form $X=\frac{\chi}{2} I_{2}$ in $\mathrm{SU}(2)$ case. Under the metric ansatz eq. (2.9), the equation of motion for $\chi$ would be

$$
\chi^{\prime \prime}+\left(\frac{-3}{z}-\Phi^{\prime}+\frac{q^{\prime}}{2 q}+\frac{h^{\prime}}{h}+\frac{f^{\prime}}{f}\right) \chi^{\prime}-\frac{1}{z^{2} f} \partial_{\chi} V(\chi)=0,
$$

where we have defined $V(\chi)=\operatorname{Tr} V_{X}$.

If we look into the above equation, magnetic effects enter this equation through three metric functions $f, q, h$. Since $\Phi, q, h$ only appears in the $\chi^{\prime}$ terms, we can recombine them and define an effective dilaton $\phi=\Phi-\log (\sqrt{q} h)$. Using $\phi$, eq. (3.2) becomes

$$
\chi^{\prime \prime}+\left(\frac{-3}{z}-\phi^{\prime}+\frac{f^{\prime}}{f}\right) \chi^{\prime}-\frac{1}{z^{2} f} \partial_{\chi} V(\chi)=0 .
$$

When $B=0$, we have $q=h \equiv 1$ and the above equation reduces to the form

$$
\chi^{\prime \prime}+\left(\frac{-3}{z}-\Phi^{\prime}+\frac{f^{\prime}}{f}\right) \chi^{\prime}-\frac{1}{z^{2} f} \partial_{\chi} V(\chi)=0 .
$$

just the same as the one without magnetic field as shown in $[67,68]$. In the numerical analysis of $[67,68]$, it is found that a large negative part of dilaton field would enhance the chiral condensate (See figure 8 and figure 9 in $[67,68]$ ). Therefore, if the extra term $-\log (\sqrt{q} h)$ is negative, we would expect a magnetic catalysis effects, while if it is positive we will expect the IMC. Since we only consider in the small $B$ region for the perturbative solution, we could expand $-\log (\sqrt{q} h)$ in powers of $B$, and the leading term is $z^{8} \log ^{2}(\mu z) B^{4}>0$. In this sense, we would expect an IMC effect in small $B$ and large $T$ region, while the qualitative behavior in other region requires a full solution. The dilaton field could be dual to the coupling constant. In some sense, the magnetic field dependent effective dilaton field works like a coupling constant running with magnetic field. Thus, if there is IMC in this scenario, in some sense it supports the studies in refs. $[36,40]$. But here, we emphasize that the above analysis is a quite rough one. We have not considered the changes of $f(z)$, so in the next sections we will check it numerically. 


\subsection{Inverse magnetic catalysis in the chiral limit}

It is easy to check that the UV structure of the solution of $\chi$ still has the form

$$
\chi=m_{q} \zeta z+\ldots+\frac{\sigma}{\zeta} z^{3}+\ldots,
$$

with $m_{q}, \sigma$ two integral constants of eq. (3.2), standing for quark mass and chiral condensate respectively, and $\zeta=\sqrt{3} /(2 \pi)$ a normalization constant [78]. As explained in [49, 67, 68], there is an additional boundary condition $f^{\prime} \chi^{\prime}-e^{2 A_{s}} \partial_{\chi} V(\chi)=0$ at the horizon $z=z_{h}$ where $f\left(z_{h}\right)=0$. This IR boundary condition would make $\sigma$ be a function of quark mass, temperature and magnetic field. Using the numerical method described in [67,68], we can extract $\sigma$ for given $m_{q}, T, B$ numerically. As an extension of the previous work, we still take the dilaton of the form

$$
\Phi(z)=-\mu_{1}^{2} z^{2}+\left(\mu_{1}^{2}+\mu_{0}^{2}\right) z^{2} \tanh \left(\mu_{2}^{2} z^{2}\right),
$$

which is of negative quadratic form $-\mu_{1}^{2} z^{2}$ in the UV region and positive quadratic form $\mu_{0}^{2} z^{2}$ in the IR region. Here, in principle, the dilaton field could depend on the magnetic filed. To determine the exact dependent behavior, we need to solve the Einstein-DilatonMaxwell coupled system, which is quite complicated. We will leave this hard task to the future and only consider the simple assumption in eq. (3.6). The scalar potential $V(\chi)$ will also be kept as

$$
V(\chi)=-\frac{3}{2} \chi^{2}+v_{4} \chi^{4}
$$

$\mu_{0}$ is related to the Regge slope of light meson spectral and is fixed to be $\mu_{0}=0.43 \mathrm{GeV}$. In $[67,68], \mu_{1}, \mu_{2}, v_{4}$ are fixed to be $\mu_{1}=0.830 \mathrm{GeV}, \mu_{2}=0.176 \mathrm{GeV}, \mathrm{v}_{4}=8$, with which the chiral phase transition temperature and the value of chiral condensate in vacuum are set to be around $150 \mathrm{MeV}$ and $(327 \mathrm{MeV})^{3}$, in coincidence with lattice results. In $[67,68]$, we displayed the dependence of chiral condensate on the form of dilaton field and showed that a larger negative part of the dilaton field would produce a larger chiral condensate with the same scalar potential. In the latter calculation, we will stick to the same parameter values.

Firstly, we check the chiral limit case by taking $m_{q}=0$, and extract $\sigma$ for different temperatures and magnetic fields. The numerical results are shown in figure 3 . In this figure, we plot the temperature dependent chiral condensate for $B=0,0.05,0.1,0.15,0.2,0.23 \mathrm{GeV}^{2}$. In Panel.(a), we give the results for small $B$. For each value of $B$, we estimates the errors by varying $\mu$ from $0.25 \mathrm{GeV}$ to $1 \mathrm{GeV}$. Then we see that at temperature range from $60 \mathrm{MeV}$ to $160 \mathrm{MeV}$, the three lines for different value of $\mu$ almost overlap, which shows that in the case of small magnetic field, the perturbative expansion is good approximation at this temperature region. Also, we see that at the same temperature, the chiral condensate decreases when $B$ increases. Furthermore, we could see that for each $B$, a characteristic second order phase transition appears and the transition temperature decreases with increasing $B$, showing a kind of IMC effect. Then, in Panel.(b), we give the results for larger $B$. We could see that when $B$ is larger than $0.15 \mathrm{GeV}^{2}$, the differences between lines for different values of $\mu$ become large even near the transition point, which confirms our analytical analysis that at large $B$ the perturbative expansion would not be good approximation anymore. 

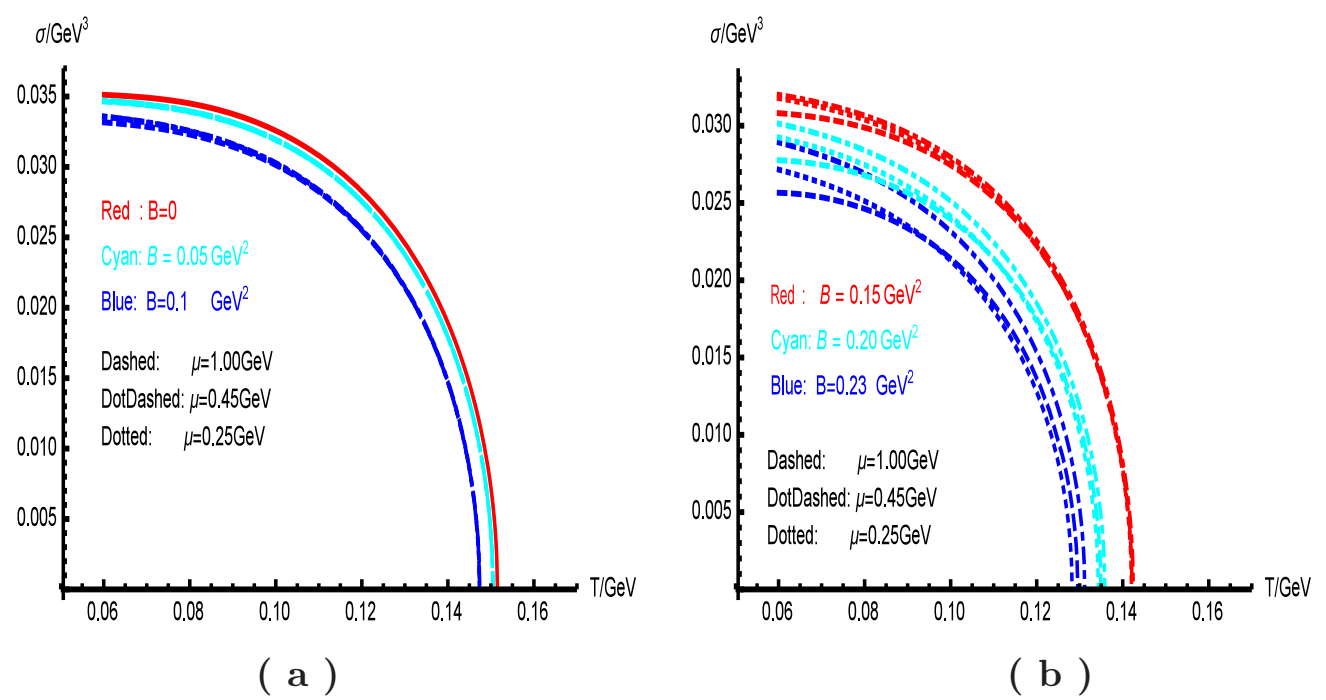

Figure 3. $\sigma$ as a function of the temperature $T$ for different values of magnetic field $B=$ $0,0.05,0.1,0.15,0.2,0.23 \mathrm{GeV}^{2}$, when $m_{q}=0, v_{4}=8, \mu_{0}=0.430 \mathrm{GeV}, \mu_{1}=0.830 \mathrm{GeV}, \mu_{2}=$ 0.176GeV. Panel.(a) gives the results for $B=0,0.05,0.1 \mathrm{GeV}^{2}$ in red, cyan, blue lines respectively. Panel.(b) gives the results for $B=0.15,0.2,0.23 \mathrm{GeV}^{2}$ in red, cyan, blue lines respectively. Both in Panel.(a) and (b), the dashed, dotdashed, dotted lines represent $\mu=1.0,0.45,0.25 \mathrm{GeV}$ respectively. For $B=0$, there is no $\mu$ dependence, and it is plotted in solid red line.

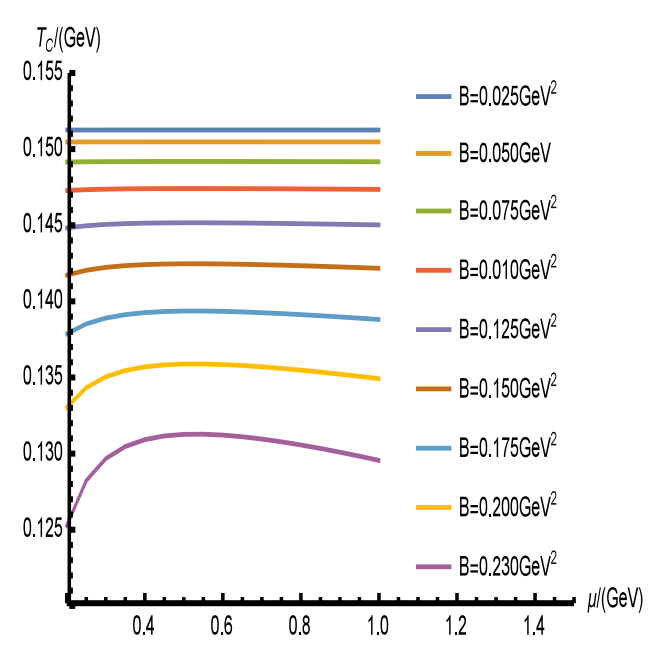

( a )

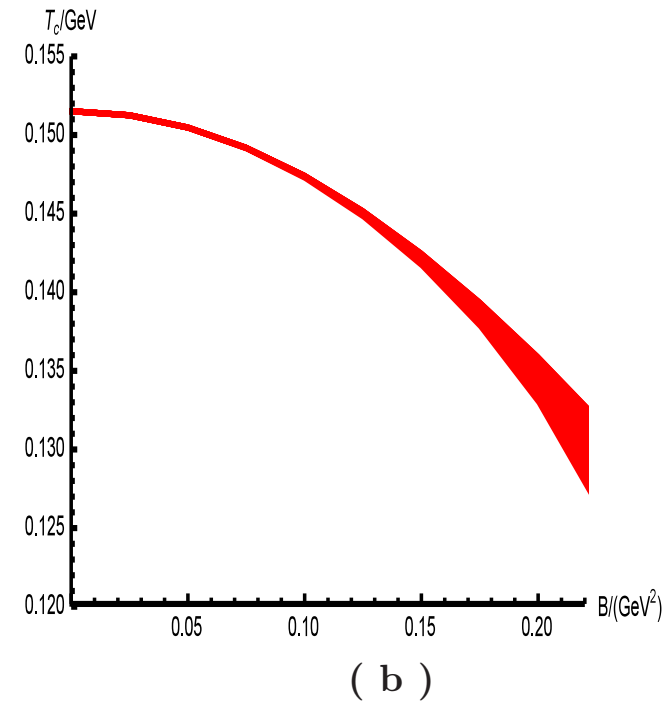

( b )

Figure 4. The critical temperature $T_{c}$ as a function of the magnetic field $B$ in the chiral limit $m_{q}=0$, when $v_{4}=8, \mu_{0}=0.430 \mathrm{GeV}, \mu_{1}=0.830 \mathrm{GeV}, \mu_{2}=0.176 \mathrm{GeV}$. In the left panel, from top to bottom, the lines stand for $B=0,0.025,0.05,0.075,0.100,0.125,0.150,0.175,0.200,0.23 \mathrm{GeV}^{2}$ respectively. In the right panel, we show how $T_{c}$ changes with $B$. In low $B$, the errors due to the uncertainty of $\mu$ are quite small. In this range decreasing behavior of $T_{c}$ with increasing $B$ are reliable. In large $B$ region, full numerical solutions are necessary to get the correct behavior of $T_{c}$. 
As can be seen in figure 3, even with finite magnetic field, chiral phase transition remains second order in chiral limit. The phase transition point is well defined and we can extract the exact transition temperature easily by numerical calculation. So we also give the $\mu$ dependent transition temperature for several values of magnetic field in figure 4. In figure 4(a), we plot the $\mu$ dependent transition temperature for $B=0,0.025,0.05,0.075,0.100,0.125,0.150,0.175,0.200,0.23 \mathrm{GeV}^{2}$ from top to down respectively. From the plot, we could see that for small $B$ (up to $B=0.15 \mathrm{GeV}^{2}$ ), the $\mu$ dependence is very weak. In some sense, it shows that the validity of the perturbative solution in this region. In addition, we could see an obvious decreasing behavior of transition temperature with increasing $B$. To be more explicitly, we plot the $\mu$ and $B$ dependent behavior of $T_{c}$ in Panel.(b). The band in Panel.(b) comes from varying $\mu$ and could be considered as an estimate of the errors by using the perturbative expansion. In small $B$ region, we see that the band is very narrow, while in large $B$ it becomes wide. From Panel.(b), we can also see an obvious IMC effect in the small $B$ region. The qualitative behavior in the large $B$ region is unknown and needs further analysis. We will leave it in the future.

In a short summary, we find that in the range $B<0.15 \mathrm{GeV}^{2}$ (related to a physical magnetic field $\mathcal{B} \approx 0.26 \mathrm{GeV}^{2}$ ) the perturbative expansion could be good approximation even near the transition temperature. In this region, the numerical results using the perturbative expansion are reliable and it shows a characteristic IMC effect. The numerical results confirm our analysis from the behavior of $-\log (\sqrt{q} h)$ in last section, which might be related to the running behavior of coupling constant with magnetic field. Here we use the perturbative expansion in powers of $z$ following [46] as a first approximation. To be more rigorous, we also follow [48] and use the perturbative expansions in powers of $B$ to check the main conclusion. We find that it gives similar results at small $B$ and high $T$ region. For the convenience of later description, we leave this part in appendix A.

\subsection{Inverse magnetic catalysis at finite quark mass}

It is also interesting to consider the case with finite quark mass, especially near physical point. Without loss of generality, we take $m_{q}=5 \mathrm{MeV}$ as an example to show the qualitative behavior at finite quark mass. Since in last section we have seen that in the valid region of the approximated expansion, the $\mu$ dependence of the results are weak, we will fix $\mu=1 \mathrm{GeV}$ in this section and only consider the results in small $B$ region as reliable results. Taking $m_{q}=5 \mathrm{MeV}$ and solving the chiral condensate from eq. (3.2), we plot the results for different values of $B$ in figure 5. In figure 5(a), we give the temperature dependent chiral condensate for $B=0,0.1,0.2,0.3,0.4 \mathrm{GeV}^{2}$ in red, orange, green, cyan, blue solid lines respectively. From the lines, we could see that the chiral phase transition turns to be a crossover one at finite quark mass. In this case, there are no exact transition points. One possible way is to define the pseudo transition point at the location of the fastest transition rate of $\sigma$, where $|d \sigma / d T|$ takes its maximum value. To show the behavior of the pseudo transition point under finite magnetic field, in figure 5(b) we also show the results of $|d \sigma / d T|$ for different $B$. From the figures, we can also read that the chiral condensate is suppressed by magnetic field at temperature near transition point, though from the 


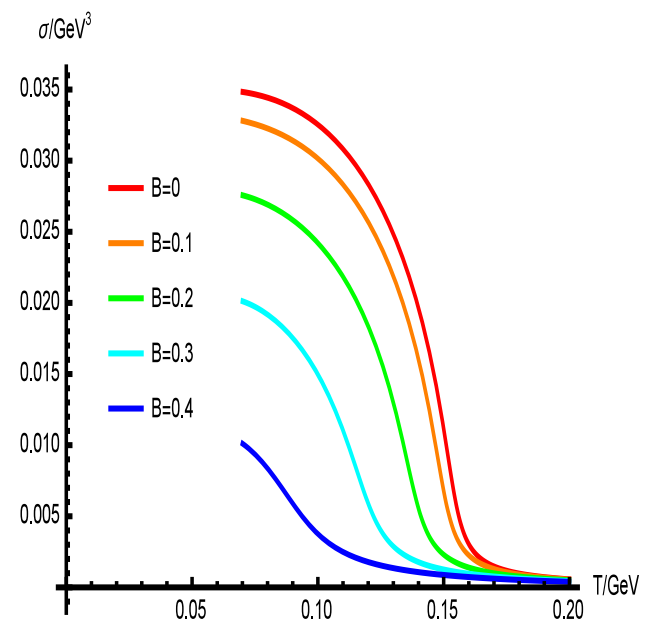

( a )

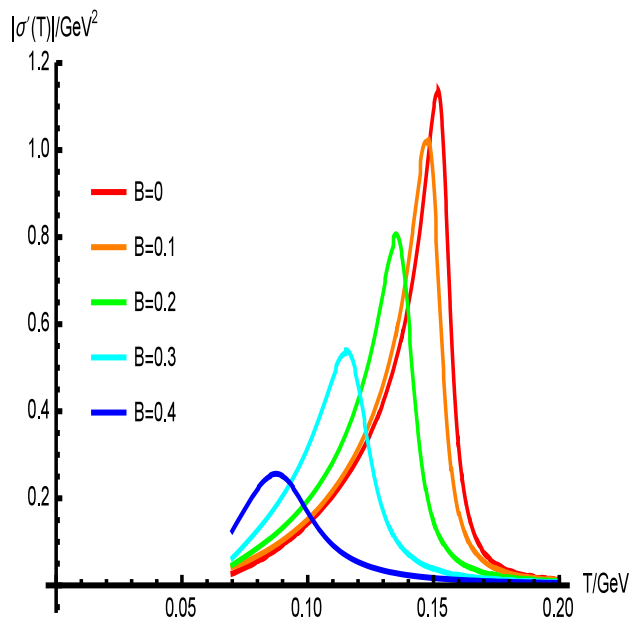

( b )

Figure 5. $\sigma$ and $\frac{d \sigma}{d T}$ as a function of temperature for different values of magnetic field $B=$ $0,0.1,0.2,0.3,0.4 \mathrm{GeV}^{2}$, when $m_{q}=5 \mathrm{MeV}, v_{4}=8, \mu_{0}=0.430 \mathrm{GeV}, \mu_{1}=0.830 \mathrm{GeV}, \mu_{2}=0.176 \mathrm{GeV}$. Panel.(a) gives results of $\sigma$ for $B=0,0.1,0.2,0.3,0.4 \mathrm{GeV}^{2}$ in red, orange, green, cyan, blue solid lines. Panel.(b) gives results of $\frac{d \sigma}{d T}$ for $B=0,0.1,0.2,0.3,0.4 \mathrm{GeV}^{2}$ in red, orange, green, cyan, blue solid lines.

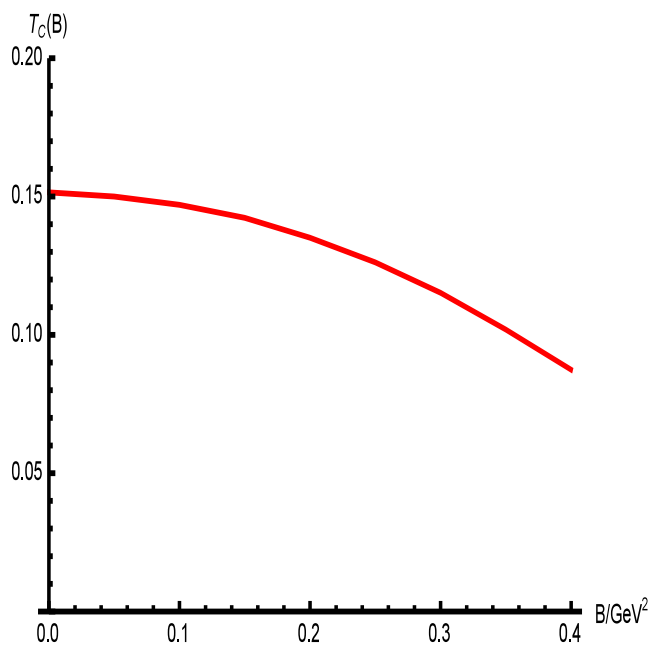

Figure 6. Critical temperature $T_{c}$ as magnetic field $B$ when $m_{q}=5 \mathrm{MeV}, v_{4}=8, \mu_{0}=$ $0.430 \mathrm{GeV}, \mu_{1}=0.830 \mathrm{GeV}, \mu_{2}=0.176 \mathrm{GeV}$ and $\mu=1 \mathrm{GeV}$.

perturbative solution we can not get the information for low temperature case. Then from figure 5(b), we see that the location of the peak in $|d \sigma / d T|$ moves towards low temperature region when increasing the magnetic field. Extracting $T_{c}$ from figure $5(\mathrm{~b})$ and plotting them in figure 6 , we could see this effects more explicitly. There the pesudo transition temperature decreases with the increasing magnetic field. Though in figure 6 we plot the figure in a wide range of $B$, we should keep in mind that the reliable results are only in small $B$ range. In large $B$ range, it is still unknown whether the transition temperature 
will decrease or increase with $B$. To get the information, a full solution with magnetic field is required, which will be done in the near future.

Therefore, we have seen inverse magnetic catalysis effect in the modified soft-wall AdS/QCD model both in chiral limit and finite quark mass cases. Mathematically, it is mainly because the extra terms in the effective dilaton field $\phi$ is positive. We have checked the arguments in previous section numerically, and we emphasize that the positivity of this extra term is guaranteed by the Einstein-Maxwell action other than model dependent effect. The dilaton field could be dual to the coupling constant [69, 79]. In some sense, the origin of IMC effect in the holographic scenario might be attribute to the correct running behavior of coupling constant, which is similar to the studies in refs. [36, 40].

\section{Conclusion and discussion}

The framework of soft-wall AdS/QCD has been widely tested from hadron physics and relevant phenomena. It is also very convenient to consider current quark mass, chiral condensate and chiral phase transition. Thus, it is very interesting to investigate magnetic effects on chiral phase transition within the framework of soft-wall AdS/QCD. The recent study in [48] shows that there are no inverse magnetic catalysis effect in the original softwall model. However, as pointed out in $[67,68]$, the original soft-wall model with a pure quadratic dilaton filed can not describe chiral phase transition well. So we consider the modified model with a dilaton field negative at ultraviolet region and positive at infrared region and an extra quartic term in the scalar potential, which has been shown to give well description on chiral phase transition in [67, 68].

To introduce magnetic field in the soft-wall model, we follow the strategy in $[46,48]$ and consider the magnetized metric solved from Einstein-Maxwell system. We try to work out the magnetized background geometry from the Einstein-Maxwell system. Due to the complexity of the equation of motion, we use the leading perturbative expansion instead of the full solution. We show that when $B$ is very small, the perturbative expansion has good convergency and can be good approximation to the full solution, while when $B$ becomes large the perturbative expansion turns to be unreliable.

Then we embed the magnetized background metric into the modified soft-wall model. We solve the magnetic field dependent chiral condensate both in chiral limit and at finite quark mass. To estimate the errors by using the perturbative solution, we vary $\mu$ from $0.2 \mathrm{GeV}$ to $1 \mathrm{GeV}$. From figure 3 , we see that when $B$ is smaller than $0.15 \mathrm{GeV}^{2}$, the results would not depend on $\mu$ sensitively in the relevant region, showing that the perturbative solution could be considered as good approximation, while when $B$ is large it can not. In reliable region, we find that the phase transition remains a second order one in chiral limit, while at finite quark mass it turns to be a crossover one. Furthermore, near the transition temperature, we find that both in chiral limit and at finite quark mass chiral condensate is suppressed by magnetic field and the transition temperature would decrease with increasing of magnetic field, showing a kind of inverse magnetic catalysis effect. From an rough analytic analysis in section 3 , we could see the main reason for the IMC effect in the holographic model is that magnetic field causes an effective term $-\log (\sqrt{q} h)$ in the 
dilaton field $\phi$. We also note that this extra term would not depend on the dilaton field in the current scenario and the IMC effect might be quite general in such a scenario. After including the full analysis, this effect would still be there, but whether there is other terms causing opposite effect still needs further studies. Furthermore, since the dilaton field could be dual to the coupling constant, this result might support the study in refs. [36, 40] that the effective running of the coupling constant with $B$ might cause the IMC effect. In the current stage, we also neglect the back-reaction from $X$ field. Thus, the effects on IMC from quark sector are neglected. In a more consistent scenario, when including the back-reaction of the quark sector, one might be able to analyze sea quarks' effect as well as the gluons'.

In this work, we have not considered the back-reaction of dilaton field to the background metric, and the dilaton field does not depend on magnetic field. In principle, if we consider a more general Einstein-Dilaton-Maxwell system, we might get more consistent results. In the more consistent study, we still have degrees of freedom like the dilaton potential and the interaction between the fields. By tuning these terms carefully, it is possible to obtained similar qualitative results. Also, since we use the perturbative expansion only, the large magnetic field $B$ and small temperature $T$ results are unreliable. We will leave the more consistent scenario and the full solution in the future.

\section{Acknowledgments}

The authors thank Kaddour Chelabi, Zheng Fang, Stefano Ivo Finazzo, Song He and Yue-Liang $\mathrm{Wu}$ for valuable discussions. D.L. is supported by the NSFC under Grant No. 11647141. M.H. is supported by the NSFC under Grants No. 11621131001, and No. 11275213. Y.Y. and P-H.Y. are supported by the Ministry of Science and Technology (NSC 101-2112-M-009-005). This work is also partly supported by China Postdoctoral Science Foundation(2015M580136).

\section{A Perturbative solutions in powers of $B$}

In the above study, we follow [46] and use the magnetized perturbative solutions in powers of $z$ showing in eqs. (2.18)-(2.20) to extract the magnetic field dependent chiral condensate. We expect that when temperature is high and magnetic field is small the perturbative solutions could give good approximation to the full solutions. The convergency of the results at high temperature and small magnetic field in some sense has confirmed this surmise. However, to be more rigorous, here, we will follow [48] and use the perturbative solutions in powers of $B$ to check the conclusion again.

From the eqs. (2.6)-(2.9), when $B=0$, the solutions should reduce to the AdS-SW black hole solutions

$$
\begin{aligned}
& f(z)=1-\frac{z^{4}}{z_{h}^{4}}, \\
& q(z)=1 \\
& h(z)=1 .
\end{aligned}
$$


Thus, when $B$ is not very large, one can assume that the solutions would be of the following form to the order of $B^{2}$

$$
\begin{aligned}
& f(z)=1-\frac{z^{4}}{z_{h}^{4}}+B^{2} f_{2}(z)+O\left(B^{4}\right), \\
& q(z)=1+\frac{8}{3} B^{2} q_{2}(z)+O\left(B^{4}\right), \\
& h(z)=1-\frac{4}{3} B^{2} h_{2}(z)+O\left(B^{4}\right) .
\end{aligned}
$$

Inserting the above form to eqs. (2.6)-(2.9) and expanding the equations to powers of $B^{2}$, after simplification, one would reach

$$
\begin{aligned}
f_{2}^{\prime}(z)-\frac{4}{z} f_{2}(z)-\frac{2 z^{3}}{3} & =0, \\
q_{2}^{\prime \prime}(z)-\frac{3+z^{4} / z_{h}^{4}}{z\left(1-z^{4} / z_{h}^{4}\right)} q_{2}^{\prime}(z)-\frac{z^{2}}{1-z^{4} / z_{h}^{4}} & =0, \\
h_{2}(z) & =q_{2}(z) .
\end{aligned}
$$

Requiring $f_{2}(0)=q_{2}(0)=h_{2}(0)=0$, one can solve the above equations and get the perturbative solutions to the order of $B^{2}$ as [48]

$$
\begin{aligned}
& f(z)=1-\frac{z^{4}}{z_{h}^{4}}+\frac{2}{3} B^{2} z^{4} \log \left(\frac{z}{z_{d}}\right)+O\left(B^{4}\right), \\
& q(z)=1+\frac{B^{2} z_{h}^{4}}{6}\left(\operatorname{Li}_{2}\left(1-\frac{z^{4}}{z_{h}^{4}}\right)-\frac{\pi^{2}}{6}\right)+O\left(B^{4}\right), \\
& h(z)=1-\frac{B^{2} z_{h}^{4}}{12}\left(\operatorname{Li}_{2}\left(1-\frac{z^{4}}{z_{h}^{4}}\right)-\frac{\pi^{2}}{6}\right)+O\left(B^{4}\right),
\end{aligned}
$$

with $\operatorname{Li}_{n}(z) \equiv \sum_{k=1}^{\infty} \frac{z^{k}}{k^{n}}$ the polylogarithm functions and $z_{d}$ an integral constant. When $n=2, \partial_{z} \operatorname{Li}_{2}\left(1-\frac{z^{4}}{z_{h}^{4}}\right)=\frac{4 z^{3} \log \left(z^{4} / z_{h}^{4}\right)}{z_{h}^{4}\left(1-z^{4} / z_{h}^{4}\right)}$ and $\operatorname{Li}_{2}(1)=\frac{\pi^{2}}{6}$. Here, we take the integral constants just like [48] and as in [48], we will take $z_{d} \approx 1 \mathrm{GeV}^{-1}$ later. We note that in eq. (A.10), $z_{h}$ is not the exact horizon where $f\left(z_{h}\right)$ should be zero. As an approximation, we can solve $f\left(z_{H}\right)=0$ and get $z_{h}^{4}=z_{H}^{4} /\left(1+\frac{2}{3} z_{H}^{4} B^{2} \log \left(z_{H} / z_{d}\right)\right)$.

We can insert the above perturbative solutions eqs. (A.10)-(A.12) into eq. (3.2) and study the magnetic effects on chiral phase transition. Before we start numerical analysis, again, we can try to get information from the effective dilaton $\phi^{\prime}=\Phi-\log (\sqrt{q} h)$. It is easy to see that up to order of $B^{2},-\log (\sqrt{q} h)=\frac{8}{3} B^{4} q_{2}^{2}(z)>0$ and according the studies in $[67,68]$, an inverse magnetic catalysis effect might be expected. Then we try to whether this idea is correct or not numerically. Here we only consider the massless case. The results are shown in figure 7. In figure 7(a), we see that under the background eqs. (A.10)(A.12) chiral phase transition remains second order in chiral limit. The qualitative results are similar to figure 3(a), the one under background eqs. (2.18)-(2.20). Again, we see that chiral condensate is suppressed by magnetic field, in coincidence with our analysis from the behavior of $\phi^{\prime}$. Furthermore, we give magnetic field $B$ dependent transition 


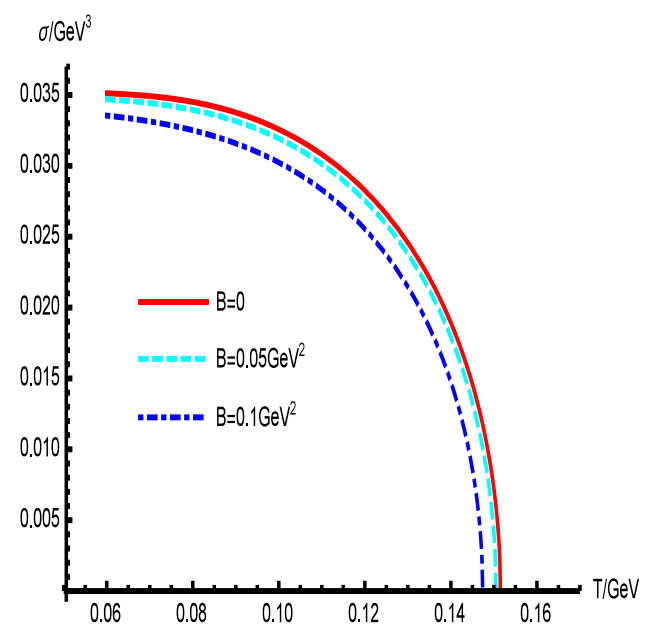

( a )

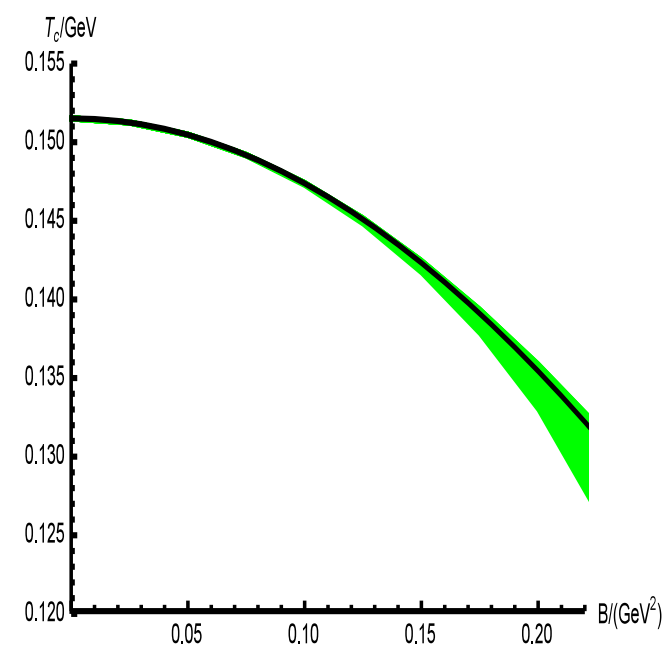

( b )

Figure 7. Chiral phase transition under perturbative background eqs. (A.10)-(A.12). Panel.(a) gives chiral condensate $\sigma(T)$ as functions of $T$ when $B=0,0.05,0.10 \mathrm{GeV}^{2}$ and $m_{q}=0$. In Panel.(b), the black solid line gives the second order phase transition temperature $T_{c}$ as a function of $B$ under the perturbative background eqs. (A.10)-(A.12). The green band are just the one under perturbative background eqs. (2.18)-(2.20).

temperature in the solid black line in figure 3(b). From this figure, we could see that the transition temperature decrease with the increasing of $B$ and at small $B$ the results are in coincidence with that under the background eqs. (2.18)-(2.20). In this way, we can trust that both the perturbative expansion eqs. (2.18)-(2.20) and eqs. (A.10)-(A.12) could give good approximation in describing chiral phase transition at small magnetic field and an inverse magnetic catalysis will happen in small magnetic field.

Open Access. This article is distributed under the terms of the Creative Commons Attribution License (CC-BY 4.0), which permits any use, distribution and reproduction in any medium, provided the original author(s) and source are credited.

\section{References}

[1] T. Vachaspati, Magnetic fields from cosmological phase transitions, Phys. Lett. B 265 (1991) 258 [INSPIRE].

[2] K. Enqvist and P. Olesen, On primordial magnetic fields of electroweak origin, Phys. Lett. B 319 (1993) 178 [hep-ph/9308270] [INSPIRE].

[3] V. Skokov, A. Yu. Illarionov and V. Toneev, Estimate of the magnetic field strength in heavy-ion collisions, Int. J. Mod. Phys. A 24 (2009) 5925 [arXiv:0907.1396] [INSPIRE].

[4] V. Voronyuk, V.D. Toneev, W. Cassing, E.L. Bratkovskaya, V.P. Konchakovski and S.A. Voloshin, (Electro-)magnetic field evolution in relativistic heavy-ion collisions, Phys. Rev. C 83 (2011) 054911 [arXiv: 1103.4239] [INSPIRE].

[5] A. Bzdak and V. Skokov, Event-by-event fluctuations of magnetic and electric fields in heavy ion collisions, Phys. Lett. B 710 (2012) 171 [arXiv:1111.1949] [INSPIRE]. 
[6] W.-T. Deng and X.-G. Huang, Event-by-event generation of electromagnetic fields in heavy-ion collisions, Phys. Rev. C 85 (2012) 044907 [arXiv: 1201.5108] [INSPIRE].

[7] S.P. Klevansky and R.H. Lemmer, Chiral symmetry restoration in the Nambu-Jona-Lasinio model with a constant electromagnetic field, Phys. Rev. D 39 (1989) 3478 [INSPIRE].

[8] K.G. Klimenko, Three-dimensional Gross-Neveu model in an external magnetic field, Theor. Math. Phys. 89 (1992) 1161 [Teor. Mat. Fiz. 89 (1991) 211] [InSPIRE].

[9] V.P. Gusynin, V.A. Miransky and I.A. Shovkovy, Dimensional reduction and catalysis of dynamical symmetry breaking by a magnetic field, Nucl. Phys. B 462 (1996) 249 [hep-ph/9509320] [INSPIRE].

[10] I.A. Shovkovy, Magnetic catalysis: a review, Lect. Notes Phys. 871 (2013) 13 [arXiv: 1207.5081] [INSPIRE].

[11] I.A. Shushpanov and A.V. Smilga, Quark condensate in a magnetic field, Phys. Lett. B 402 (1997) 351 [hep-ph/9703201] [INSPIRE].

[12] N.O. Agasian and I.A. Shushpanov, The quark and gluon condensates and low-energy QCD theorems in a magnetic field, Phys. Lett. B 472 (2000) 143 [hep-ph/9911254] [INSPIRE].

[13] J. Alexandre, K. Farakos and G. Koutsoumbas, Magnetic catalysis in $Q E D_{3}$ at finite temperature: beyond the constant mass approximation, Phys. Rev. D 63 (2001) 065015 [hep-th/0010211] [INSPIRE].

[14] N.O. Agasian, Chiral thermodynamics in a magnetic field, Phys. Atom. Nucl. 64 (2001) 554 [Yad. Fiz. 64 (2001) 608] [hep-ph/0112341] [INSPIRE].

[15] T.D. Cohen, D.A. McGady and E.S. Werbos, The chiral condensate in a constant electromagnetic field, Phys. Rev. C 76 (2007) 055201 [arXiv:0706.3208] [INSPIRE].

[16] R. Gatto and M. Ruggieri, Dressed Polyakov loop and phase diagram of hot quark matter under magnetic field, Phys. Rev. D 82 (2010) 054027 [arXiv: 1007.0790] [INSPIRE].

[17] R. Gatto and M. Ruggieri, Deconfinement and chiral symmetry restoration in a strong magnetic background, Phys. Rev. D 83 (2011) 034016 [arXiv: 1012.1291] [INSPIRE].

[18] A.J. Mizher, M.N. Chernodub and E.S. Fraga, Phase diagram of hot QCD in an external magnetic field: possible splitting of deconfinement and chiral transitions, Phys. Rev. D 82 (2010) 105016 [arXiv:1004.2712] [INSPIRE].

[19] K. Kashiwa, Entanglement between chiral and deconfinement transitions under strong uniform magnetic background field, Phys. Rev. D 83 (2011) 117901 [arXiv:1104.5167] [INSPIRE].

[20] S.S. Avancini, D.P. Menezes, M.B. Pinto and C. Providencia, The QCD critical end point under strong magnetic fields, Phys. Rev. D 85 (2012) 091901 [arXiv:1202.5641] [INSPIRE].

[21] J.O. Andersen, Thermal pions in a magnetic background, Phys. Rev. D 86 (2012) 025020 [arXiv: 1202.2051] [INSPIRE].

[22] D.D. Scherer and H. Gies, Renormalization group study of magnetic catalysis in the $3 d$ Gross-Neveu model, Phys. Rev. B 85 (2012) 195417 [arXiv:1201.3746] [INSPIRE].

[23] P.V. Buividovich, M.N. Chernodub, E.V. Luschevskaya and M.I. Polikarpov, Numerical study of chiral symmetry breaking in non-Abelian gauge theory with background magnetic field, Phys. Lett. B 682 (2010) 484 [arXiv:0812.1740] [INSPIRE]. 
[24] V.V. Braguta, P.V. Buividovich, T. Kalaydzhyan, S.V. Kuznetsov and M.I. Polikarpov, The chiral magnetic effect and chiral symmetry breaking in $\mathrm{SU}(3)$ quenched lattice gauge theory, Phys. Atom. Nucl. 75 (2012) 488 [arXiv:1011.3795] [INSPIRE].

[25] M. D'Elia, S. Mukherjee and F. Sanfilippo, QCD phase transition in a strong magnetic background, Phys. Rev. D 82 (2010) 051501 [arXiv:1005.5365] [INSPIRE].

[26] M. D'Elia and F. Negro, Chiral properties of strong interactions in a magnetic background, Phys. Rev. D 83 (2011) 114028 [arXiv:1103.2080] [inSPIRE].

[27] E.M. Ilgenfritz, M. Kalinowski, M. Muller-Preussker, B. Petersson and A. Schreiber, Two-color QCD with staggered fermions at finite temperature under the influence of a magnetic field, Phys. Rev. D 85 (2012) 114504 [arXiv: 1203.3360] [INSPIRE].

[28] G.S. Bali et al., The QCD phase diagram for external magnetic fields, JHEP 02 (2012) 044 [arXiv:1111.4956] [INSPIRE].

[29] G.S. Bali, F. Bruckmann, G. Endrodi, Z. Fodor, S.D. Katz and A. Schafer, QCD quark condensate in external magnetic fields, Phys. Rev. D 86 (2012) 071502 [arXiv:1206.4205] [INSPIRE].

[30] V.G. Bornyakov, P.V. Buividovich, N. Cundy, O.A. Kochetkov and A. Schäfer, Deconfinement transition in two-flavor lattice $Q C D$ with dynamical overlap fermions in an external magnetic field, Phys. Rev. D 90 (2014) 034501 [arXiv:1312.5628] [INSPIRE].

[31] K. Fukushima and Y. Hidaka, Magnetic catalysis versus magnetic inhibition, Phys. Rev. Lett. 110 (2013) 031601 [arXiv:1209.1319] [inSPIRE].

[32] T. Kojo and N. Su, The quark mass gap in a magnetic field, Phys. Lett. B 720 (2013) 192 [arXiv:1211.7318] [INSPIRE].

[33] F. Bruckmann, G. Endrodi and T.G. Kovacs, Inverse magnetic catalysis and the Polyakov loop, JHEP 04 (2013) 112 [arXiv: 1303.3972] [INSPIRE].

[34] J. Chao, P. Chu and M. Huang, Inverse magnetic catalysis induced by sphalerons, Phys. Rev. D 88 (2013) 054009 [arXiv: 1305.1100] [inSPIRE].

[35] E.S. Fraga, B.W. Mintz and J. Schaffner-Bielich, A search for inverse magnetic catalysis in thermal quark-meson models, Phys. Lett. B 731 (2014) 154 [arXiv:1311.3964] [INSPIRE].

[36] M. Ferreira, P. Costa, O. Lourenço, T. Frederico and C. Providência, Inverse magnetic catalysis in the $(2+1)$-flavor Nambu-Jona-Lasinio and Polyakov-Nambu-Jona-Lasinio models, Phys. Rev. D 89 (2014) 116011 [arXiv:1404.5577] [INSPIRE].

[37] R.L.S. Farias, K.P. Gomes, G.I. Krein and M.B. Pinto, Importance of asymptotic freedom for the pseudocritical temperature in magnetized quark matter, Phys. Rev. C 90 (2014) 025203 [arXiv: 1404.3931] [INSPIRE].

[38] L. Yu, H. Liu and M. Huang, Spontaneous generation of local CP-violation and inverse magnetic catalysis, Phys. Rev. D 90 (2014) 074009 [arXiv:1404.6969] [INSPIRE].

[39] J.O. Andersen, W.R. Naylor and A. Tranberg, Inverse magnetic catalysis and regularization in the quark-meson model, JHEP 02 (2015) 042 [arXiv: 1410.5247] [INSPIRE].

[40] E.J. Ferrer, V. de la Incera and X.J. Wen, Quark antiscreening at strong magnetic field and inverse magnetic catalysis, Phys. Rev. D 91 (2015) 054006 [arXiv:1407.3503] [INSPIRE]. 
[41] B. Feng, D.-F. Hou and H.-C. Ren, Magnetic and inverse magnetic catalysis in the Bose-Einstein condensation of neutral bound pairs, Phys. Rev. D 92 (2015) 065011 [arXiv: 1412.1647] [INSPIRE].

[42] J.M. Maldacena, The large- $N$ limit of superconformal field theories and supergravity, Int. J. Theor. Phys. 38 (1999) 1113 [hep-th/9711200] [INSPIRE].

[43] S.S. Gubser, I.R. Klebanov and A.M. Polyakov, Gauge theory correlators from noncritical string theory, Phys. Lett. B 428 (1998) 105 [hep-th/9802109] [INSPIRE].

[44] E. Witten, Anti-de Sitter space and holography, Adv. Theor. Math. Phys. 2 (1998) 253 [hep-th/9802150] [INSPIRE].

[45] S.-W. Li and T. Jia, Dynamically flavored description of holographic QCD in the presence of a magnetic field, arXiv:1604.07197 [INSPIRE].

[46] K.A. Mamo, Inverse magnetic catalysis in holographic models of QCD, JHEP 05 (2015) 121 [arXiv: 1501.03262] [INSPIRE].

[47] N. Evans, C. Miller and M. Scott, Inverse magnetic catalysis in bottom-up holographic QCD, Phys. Rev. D 94 (2016) 074034 [arXiv: 1604.06307] [INSPIRE].

[48] D. Dudal, D.R. Granado and T.G. Mertens, No inverse magnetic catalysis in the QCD hard and soft wall models, Phys. Rev. D 93 (2016) 125004 [arXiv:1511.04042] [INSPIRE].

[49] A. Karch, E. Katz, D.T. Son and M.A. Stephanov, Linear confinement and AdS/QCD, Phys. Rev. D 74 (2006) 015005 [hep-ph/0602229] [INSPIRE].

[50] T. Gherghetta, J.I. Kapusta and T.M. Kelley, Chiral symmetry breaking in the soft-wall AdS/QCD model, Phys. Rev. D 79 (2009) 076003 [arXiv:0902.1998] [INSPIRE].

[51] T.M. Kelley, S.P. Bartz and J.I. Kapusta, Pseudoscalar mass spectrum in a soft-wall model of AdS/QCD, Phys. Rev. D 83 (2011) 016002 [arXiv:1009.3009] [InSPIRE].

[52] Y.-Q. Sui, Y.-L. Wu, Z.-F. Xie and Y.-B. Yang, Prediction for the mass spectra of resonance mesons in the soft-wall AdS/QCD with a modified $5 D$ metric,

Phys. Rev. D 81 (2010) 014024 [arXiv:0909.3887] [InSPIRE].

[53] Y.-Q. Sui, Y.-L. Wu and Y.-B. Yang, Predictive AdS/QCD model for mass spectra of mesons with three flavors, Phys. Rev. D 83 (2011) 065030 [arXiv: 1012.3518] [INSPIRE].

[54] Z. Fang, D. Li and Y.-L. Wu, IR-improved soft-wall AdS/QCD model for baryons, Phys. Lett. B 754 (2016) 343 [arXiv: 1602.00379] [INSPIRE].

[55] D. Li, M. Huang and Q.-S. Yan, A dynamical soft-wall holographic QCD model for chiral symmetry breaking and linear confinement, Eur. Phys. J. C 73 (2013) 2615 [arXiv: 1206.2824] [INSPIRE].

[56] D. Li and M. Huang, Dynamical holographic QCD model for glueball and light meson spectra, JHEP 11 (2013) 088 [arXiv:1303.6929] [INSPIRE].

[57] P. Colangelo, F. De Fazio, F. Giannuzzi, F. Jugeau and S. Nicotri, Light scalar mesons in the soft-wall model of AdS/QCD, Phys. Rev. D 78 (2008) 055009 [arXiv:0807.1054] [INSPIRE].

[58] S. He, S.-Y. Wu, Y. Yang and P.-H. Yuan, Phase structure in a dynamical soft-wall holographic QCD model, JHEP 04 (2013) 093 [arXiv: 1301.0385] [INSPIRE].

[59] Y. Chen and M. Huang, Two-gluon and trigluon glueballs from dynamical holography QCD, Chin. Phys. C 40 (2016) 123101 [arXiv:1511.07018] [inSPIRE]. 
[60] E. Folco Capossoli and H. Boschi-Filho, Odd spin glueball masses and the odderon Regge trajectories from the holographic hardwall model, Phys. Rev. D 88 (2013) 026010 [arXiv: 1301.4457 ] [INSPIRE].

[61] E. Folco Capossoli and H. Boschi-Filho, Glueball spectra and Regge trajectories from a modified holographic softwall model, Phys. Lett. B 753 (2016) 419 [arXiv:1510.03372] [INSPIRE].

[62] E. Folco Capossoli, D. Li and H. Boschi-Filho, Pomeron and odderon Regge trajectories from a dynamical holographic model, Phys. Lett. B 760 (2016) 101 [arXiv:1601.05114] [INSPIRE].

[63] E. Folco Capossoli, D. Li and H. Boschi-Filho, Dynamical corrections to the anomalous holographic soft-wall model: the pomeron and the odderon, Eur. Phys. J. C 76 (2016) 320 [arXiv: 1604.01647] [INSPIRE].

[64] E. D'Hoker and P. Kraus, Charged magnetic brane solutions in AdS $S_{5}$ and the fate of the third law of thermodynamics, JHEP 03 (2010) 095 [arXiv: 0911.4518] [INSPIRE].

[65] E. D'Hoker and P. Kraus, Magnetic brane solutions in AdS, JHEP 10 (2009) 088 [arXiv: 0908.3875] [INSPIRE].

[66] M. Ammon, J. Leiber and R.P. Macedo, Phase diagram of 4D field theories with chiral anomaly from holography, JHEP 03 (2016) 164 [arXiv: 1601.02125] [INSPIRE].

[67] K. Chelabi, Z. Fang, M. Huang, D. Li and Y.-L. Wu, Realization of chiral symmetry breaking and restoration in holographic QCD, Phys. Rev. D 93 (2016) 101901 [arXiv:1511.02721] [INSPIRE].

[68] K. Chelabi, Z. Fang, M. Huang, D. Li and Y.-L. Wu, Chiral phase transition in the soft-wall model of AdS/QCD, JHEP 04 (2016) 036 [arXiv:1512.06493] [INSPIRE].

[69] U. Gürsoy, E. Kiritsis, L. Mazzanti and F. Nitti, Holography and thermodynamics of $5 D$ dilaton-gravity, JHEP 05 (2009) 033 [arXiv:0812.0792] [INSPIRE].

[70] P. Colangelo, F. Giannuzzi and S. Nicotri, Holography, heavy-quark free energy and the QCD phase diagram, Phys. Rev. D 83 (2011) 035015 [arXiv:1008.3116] [INSPIRE].

[71] D. Li, S. He, M. Huang and Q.-S. Yan, Thermodynamics of deformed AdS $S_{5}$ model with a positive/negative quadratic correction in graviton-dilaton system, JHEP 09 (2011) 041 [arXiv:1103.5389] [INSPIRE].

[72] R.-G. Cai, S. He and D. Li, A hQCD model and its phase diagram in Einstein-Maxwell-dilaton system, JHEP 03 (2012) 033 [arXiv:1201.0820] [INSPIRE].

[73] D. Li, J. Liao and M. Huang, Enhancement of jet quenching around phase transition: result from the dynamical holographic model, Phys. Rev. D 89 (2014) 126006 [arXiv:1401.2035] [INSPIRE].

[74] D. Li, S. He and M. Huang, Temperature dependent transport coefficients in a dynamical holographic QCD model, JHEP 06 (2015) 046 [arXiv:1411.5332] [INSPIRE].

[75] Y. Yang and P.-H. Yuan, A refined holographic QCD model and QCD phase structure, JHEP 11 (2014) 149 [arXiv:1406.1865] [INSPIRE].

[76] Y. Yang and P.-H. Yuan, Confinement-deconfinement phase transition for heavy quarks in a soft wall holographic QCD model, JHEP 12 (2015) 161 [arXiv:1506.05930] [INSPIRE].

[77] Z. Fang, S. He and D. Li, Chiral and deconfining phase transitions from holographic QCD study, Nucl. Phys. B 907 (2016) 187 [arXiv:1512.04062] [INSPIRE]. 
[78] A. Cherman, T.D. Cohen and E.S. Werbos, The chiral condensate in holographic models of QCD, Phys. Rev. C 79 (2009) 045203 [arXiv: 0804.1096] [inSPIRE].

[79] S. He, M. Huang and Q.-S. Yan, Logarithmic correction in the deformed AdS $S_{5}$ model to produce the heavy quark potential and QCD $\beta$-function, Phys. Rev. D 83 (2011) 045034 [arXiv: 1004.1880] [INSPIRE].

[80] R. Critelli, S.I. Finazzo, M. Zaniboni and J. Noronha, Anisotropic shear viscosity of a strongly coupled non-Abelian plasma from magnetic branes, Phys. Rev. D 90 (2014) 066006 [arXiv: 1406.6019] [INSPIRE].

[81] R. Rougemont, R. Critelli and J. Noronha, Anisotropic heavy quark potential in strongly-coupled $N=4$ SYM in a magnetic field, Phys. Rev. D 91 (2015) 066001 [arXiv: 1409.0556] [INSPIRE].

[82] S.I. Finazzo, R. Critelli, R. Rougemont and J. Noronha, Momentum transport in strongly coupled anisotropic plasmas in the presence of strong magnetic fields, Phys. Rev. D 94 (2016) 054020 [arXiv: 1605.06061] [InSPIRE]. 